\title{
Ground-Based Studies of Emission-Line Variability: Recent Results for NGC 5548 and Future Plans
}

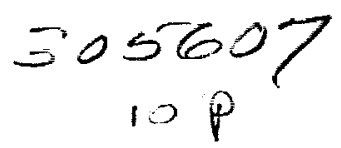

\author{
Bradley M. Peterson \\ Department of Astronomy, The Ohio State University
}

\section{Introduction}

Ever since emission-line variability was first detected in AGNs, it has been clearly understood that light-travel time effects in the broad-line region (BLR) afford a unique tool for studying the structure of these spatially unresolved regions, (see Peterson 1988 for a review). However, only within the last few years has the observational problem become well defined in terms of the sampling rates and quality of data necessary to address the problem correctly. The amount of data required to extract structural information about the BLR from the continuum and emission-line light curves is so considerable that nanof us were convinced that the most promising approach would be to combine our observational efforts. Our goal was to work together to produce a large, high-quality database which would then be released to individual investigators for more complete analysis. The cornerstone of this group effort, described in these proceedings by Clavel, was a joint NASA/ESA/SERC program to monitor the spectrum of NGC 5548 every four days with IUE from 1988 December through 1989 August (Clavel et al. 1990), A concurrent ground-based program was organized in an effort to enhance the scientific return on the project by extending the wavelength coverage and providing higher spectral resolution and signal-to-noise ratios than would be possible with IUE. It also turned out that the temporal resolution of the ground-based program was somewhat better than the temporal resolution of the $I U E$ program, and the temporal baseline is longer and continues to grow. The database and initial results are presented by Peterson et al. (1991). In this contribution, I will summarize the important results of the ground-based program, describe some additional applications of the existing data, and make a few comments on what we can do in the future to expand on this work.

\section{The NGC 5548 Optical Database}

The ground-based study of the temporal behavior of NGC 5548 differed from the concurrent $I U E$ project in that it was not a joint program undertaken with a single instrument. The basis of the ground-based program was an informal agreement that participants would obtain optical spectra, photometry, and CCD images of NGC 5548 whenever possible, although there were a few notable cases where observers devoted much of their

$$
\begin{aligned}
& \text { (NASA-CR-160433) GROUND-PASED STUUIES OF } \\
& \text { EMISSIUN-LINE VARIAAILITY: RECENT RESULTS } \\
& \text { FOR NGC } 5548 \text { ANT FUTURE PLANS fOHio State } \\
& \text { Univ.) } 10 \mathrm{p} \\
& \text { CSCL O3A }
\end{aligned}
$$

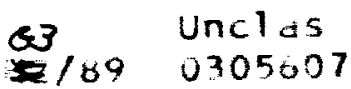


observational effort for the year to this single project. The ground-based data are not evenly spaced, as AGN observers tend to be at the telescope during dark or gray time rather than bright time, and the data are not homogeneous, as they were obtained with many different instruments in a variety of configurations.

As a first step in the analysis of these data, we have examined the spectral variations in the vicinity of the $\mathrm{H} \beta$ emission line. The principal reason for this choice is the proximity of the [O III] $\lambda \lambda 4959,5007$ narrow emission lines, which do not vary over the time scales of interest and thus can be used for absolute flux calibration if the [O III] flux is determined accurately from observations under photometric conditions. Evidence for aperture effects is seen by comparing the data obtained with different instruments - larger spectrograph entrance apertures admit relatively more [O $\mathrm{m}]$ flux from the partially resolved narrowline region as well as more starlight from the host galaxy. In practice, we find that we can apply empirically determined small nominal corrections to the data obtained with different instruments to put all of the measurements on a common flux scale.

The database for the study of continuum and $\mathrm{H} \beta$ variations in NGC 5548 consists of 177 spectra covering 129 independent dates between 1988 December 14 and 1989 October 10. Except for the final month of the campaign, when only two observations were obtained, the temporal coverage was extraordinarily good, with the average interval between observations being 3.1 days, and a median interval of only 1 day. There are no gaps in the coverage larger than 12 days, and gaps of a week or longer are rare. The typical uncertainty in the continuum measurements (at $4870 \AA$, underneath the $B \beta$ line) is $\sim 4.5 \%$, and the mean uncertainty in the $\mathrm{H} \beta$ measurements is $\sim 3.7 \%$. The continuum and emission-line light curves are shown in Fig. 1, which show that three significant continuum "events" were detected during this campaign.

\section{Phase Analysis: Cross-Correlation Results}

The primary scientific goal of this project was to determine the "lag" or phase shift between the continuum and emission-line light curves, and also to determine whether or not there is a lag between the ultraviolet and optical continuum variations. The lag between two light curves is determined by cross correlation, either by using the interpolation method (Gaskell and Sparke 1986) or the discrete correlation method (Edelson and Krolik 1988) - in this particular case, the light curves are so well sampled that it makes no difference which method is used.

Cross correlation of the ultraviolet and optical continuum light curves shows that the optical continuum variations follow those in the ultraviolet by $\sim 2$ days; various methods of assessing the uncertainty in the cross-correlation lag all seem to indicate that the formal uncertainties in the results discussed here are 2-3 days, which indicates that the ultraviolet - optical continuum lag is not significantly different from zero, and thus should not be taken too seriously.

Cross correlation of the continuum and $\mathrm{H} \beta$ light curves gives a lag of $\sim 20$ days. This result is highly significant (the correlation coefficient at the peak of the cross-correlation function is $r \approx 0.86$ ), and is significantly different from the $\sim 10$-day lag found for Ly $\alpha$ from the IUE data; direct cross-correlation of the Ly $\alpha$ and $\mathrm{H} \beta$ light curves shows that $\mathrm{H} \beta$ lags Ly $\alpha$ by $\sim 8$ days. This appears to be consistent with the $I U E$ results for other emission lines, which suggest that the high ionization lines respond more rapidly than low 


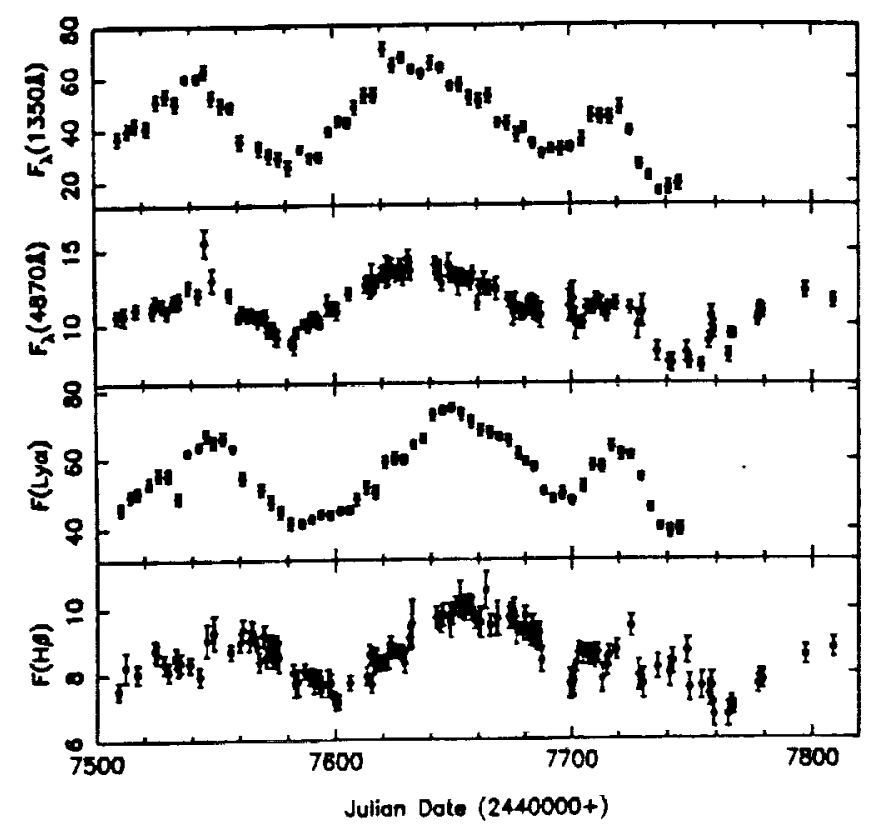

Fig. 1. The continuum light curves at $1350 \AA$ and $4870 \AA$ are shown, as well as the emission-line light curves for Ly $\alpha$ and $H \beta$. Continuum fluxes are in units of $10^{-15} \mathrm{ergs} \mathrm{s}^{-1} \mathrm{~cm}^{-2} \AA^{-1}$ and line fluxes are in units of $10^{-13} \mathrm{ergs} \mathrm{s}^{-1} \mathrm{~cm}^{-2}$ (from Peterson et al. 1991).

ionization lines to changes in the continuum flux. This has been interpreted as evidence for ionization stratification of the BLR.

\section{Continuum Amplitude Analysis: The Host Galaxy Contribution}

The absence of a phase shift between the ultraviolet and optical continuum light curves shows that at least the variable parts of the ultraviolet and optical continuum have a common origin. Further investigation of the origin of the variable continuum requires that we try to remove the non-varying or slowly varying components from the measured flux in each continuum band. Determination of the stellar contribution to the optical continuum measurements is especially important in determining the relative amplitude of variability in the optical and the ultraviolet.

Particularly useful in this regard are observations made during very faint states, as these provide firm upper limits to the flux in other components, and indeed the contributions due to underlying starlight and narrow emission lines, for example, become 


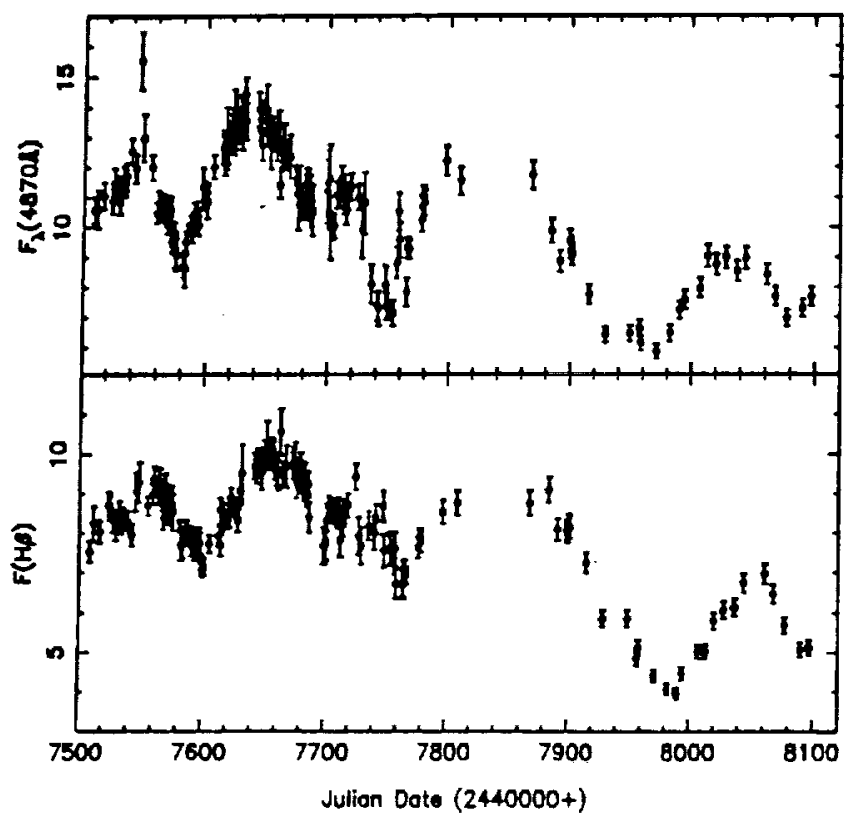

Fig. 2. The optical continuum and $\mathrm{H} \beta$ light curves for the two most recent observing seasons. Units as in Fig. 1.

relatively prominent and easier to evaluate when the nonstellar continuum and broad emission lines are weak. We were therefore very fortunate to observe NGC 5548 in its faintest recorded state during the most recent observing season. We have continued to monitor NGC 5548, and a preliminary optical light curve, so far based on Ohio State data only, is shown in Fig. 2. This light curve shows that in 1990 March - April, NGC 5548 was remarkably faint. An IUE spectrum obtained close to minimum light (Reichert, Webb, and Crenshaw 1990) shows that the nucleus was at that time only half as bright as in its previously recorded faintest state.

There are a number of ways that the host-galaxy contribution to the optical continuum can be measured, and here we describe a very simple method. As shown in Fig. 3, we plot the optical continuum flux at $5100 \AA$ versus the simultaneously measured ultraviolet flux at $1350 \AA$ (note that we have restricted this analysis to the homogeneous Ohio State data, obtained through an entrance aperture of 5 arcsec $\times 7.6$ arcsec, and we have used a more "line-free" wavelength than in the earlier analysis). If it is assumed that no other spectral components other than the variable continuum and the starlight (measurable only in the optical) contribute to these continuum bands, then the $y$-intercept of this relationship gives the starlight contribution at $5100 \AA$, and any non-linearity indicates a change in spectral index with continuum level. 


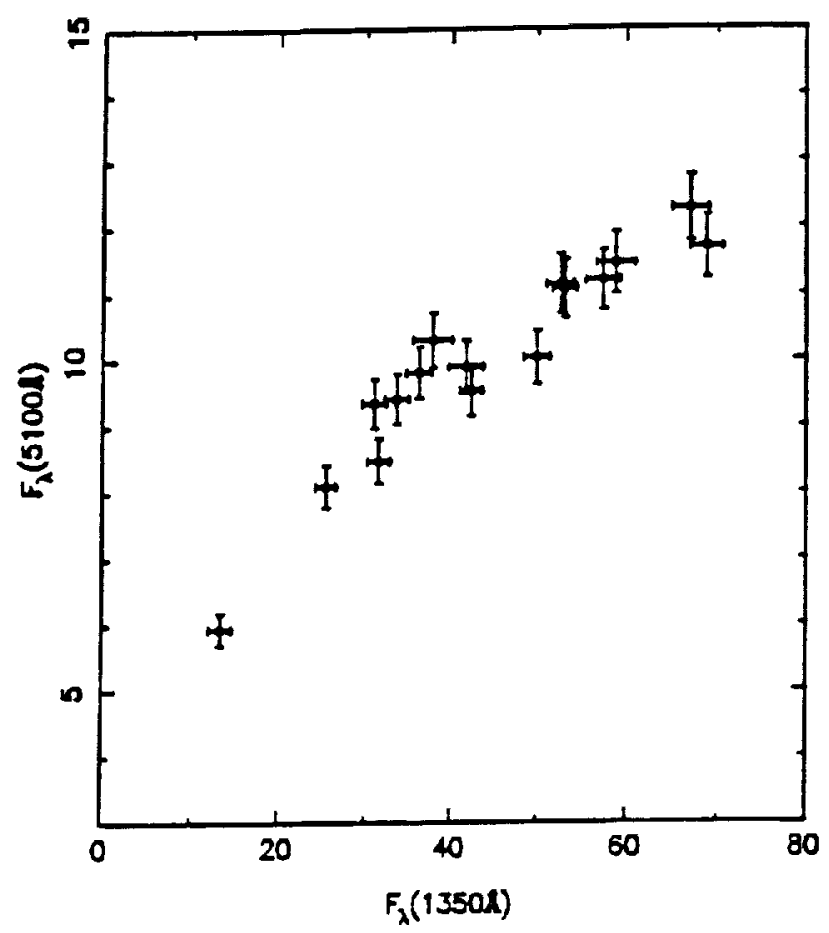

Fig. 3. The relationship between the total continuum flux at $5100 \AA$, as measured through a $5 \times 7.6$ arcsec ${ }^{2}$ aperture, and the total continum flux at $1350 \AA$. Fluxes are in units of $10^{-15}$ ergs $\mathrm{s}^{-1} \mathrm{~cm}^{-2} \AA^{-1}$.

From Fig. 3, one can see that the best-fit function to these data will have some curvature, in the sense that the uitraviolet flux increases by a larger factor than does the optical flux. In other words, the spectrum gets harder as it gets brighter. By fitting a quadratic to these data, we find that the $y$-intercept of this function is $(3.37 \pm 0.54) \times$ $10^{-15} \mathrm{ergs} \mathrm{s}^{-1} \mathrm{~cm}^{-2} \AA^{-1}$. If we adopt this as the host galaxy flux ${ }^{1}$ at $5100 \AA$ as observed through a 5 aresec $\times 7.6$ arcsec aperture and subtract this constant contribution from the optical flux measurements, we can determine a relationship between the spectral index between $1350 \AA$ and $5100 \AA$ and the continuum flux. Typically this index is about $\alpha \approx$ $0.5\left(F \propto \nu^{-\alpha}\right)$, although it increases to 0.9 in the faintest states. This result is in good agreement with the findings of Wamsteker et al. (1990).

A stringent upper limit on this quantity is provided by the total flux at minimum light, $F_{\lambda}=$ $5.46 \times 10^{-15}$ ergs s$^{-1} \mathrm{~cm}^{-2} A^{-1}$. 


\section{The $\mathbf{H} \boldsymbol{\beta}$ Transfer Function and Emissivity Distribution}

Because the sampling grid is very tight and the amplitude of variation is large relative to the noise level, it is possible to solve the integral equation which describes the response of the emission line to continuum variations, i.e.,

$$
L(t)=\int_{-\infty}^{\infty} \Psi(t-\tau) C(\tau) d \tau
$$

where $C(t)$ and $L(t)$ are the continuum and emission-line light curves, respectively, and $\Psi(t)$ is the transfer function. The transfer function is essentially the emissivity-weighted geometrical distribution of the line-emitting gas as a function of time delay as seen by an external observer (Blandford and McKee 1982). A first attempt at solving for $\Psi(t)$ has been made by Horne, Weish, and Peterson (1990) by using the maximum entropy method. The resulting transfer function for $\mathrm{H} \beta$ is shown in Fig. 4. The remarkable thing about this function is that it approaches zero near zero lag; by contrast, the transfer function for a thin spherical shell of radius $R$ is a rectangular function which is non-zero in the range $0 \leq t \leq 2 R / c$. The lack of immediate response of the $\mathrm{B} \beta$ emission line to continuum variations implies that the emissivity along our line of sight to the continuum source is very low. This points to a flattened geometry which we are observing more or less close to pole-on. Further discussion appears in these proceedings in the paper by Horne.

\section{Work in Progress}

At this point, we have only scratched the surface of the information available in the NGC 5548 database. Some important additional studies which are currently underway include:

- Determination of the spectral energy distribution over a larger wavelength range. This includes attempting to refine the model for the underlying starlight distribution.

- Determination of the velocity field and kinematics of the BLR. As described in these proceedings, Kollatschny and others are examining the high- $S / N$, high-resolution optical spectra to look for line-profile changes. Crenshaw and Blackwell (1990) have examined the behavior of the $C$ IV $\lambda 1549$ line during the period at the end of the $I U E$ campaign when the continuum and the lines decreased rapidly in flux. On the basis of their analysis, they conclude that the red side of the line profile follows the continuum more rapidly than the blue side, which argues for net infall of the BLR gas. Unfortunately, we do not see any such gross effect when we analyze all of the $\mathrm{C}$ iv $\lambda 1549$ data for the campaign, so it is not yet clear what this result means. Efforts are also being made to recover the velocity-dependent transfer function from the optical data.

- Measurement of the variability of the blue bump. The mean ultraviolet-optical NGC 5548 spectrum of Wamsteker et al. (1990) indicates that $\sim 1 / 3$ of the emission from the BLR comes out in the "small bump", and obviously this important source of cooling needs to be better understood before detailed photoionization models can be attempted.

- Measurement of other optical emission lines. Additional important broad lines which can be examined in the optical spectra include other Balmer series lines, He II $\lambda 4686$, 


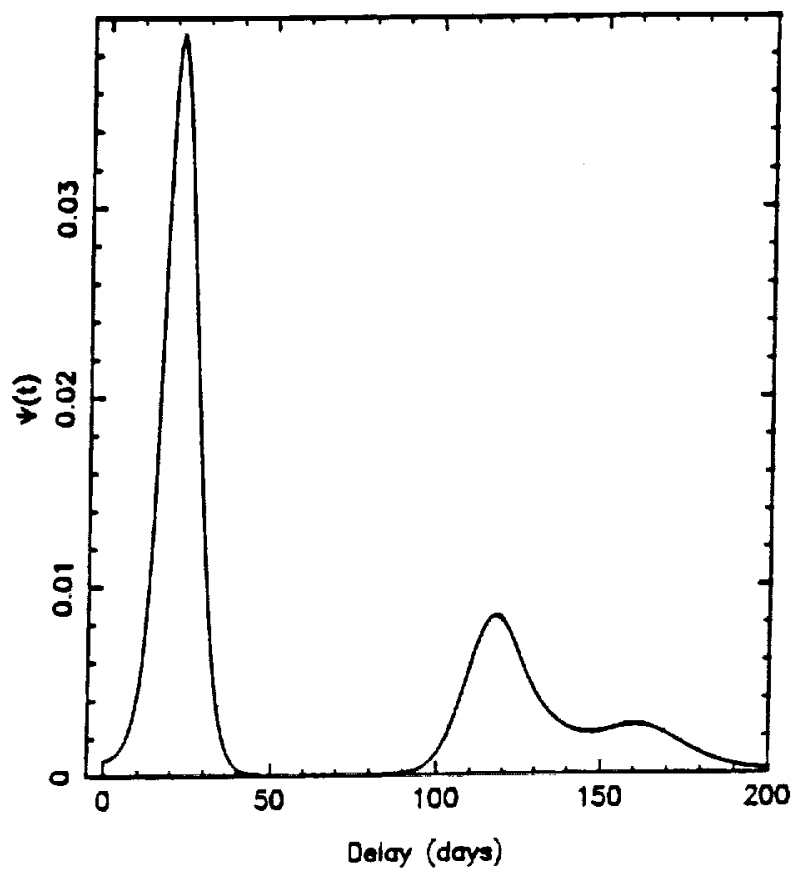

Fig. 4. The transfer function for the $H \beta$ emission line (from Horne, Welsh, and Peterson 1990).

He I $\lambda 5876$, and the optical Fe II blends. These will require more effort to analyze as the flux calibration away from [O III] $\lambda \lambda 4959,5007$ must be checked very carefully, and some of these lines are very low contrast features which are often badly blended with other lines.

\section{Impact on Future Studies}

It is clear from the success of the NGC 5548 project that similar projects can and should be pursued in the future. Therefore, I think it is instructive to reflect briefly on some of the reasons that this particular effort was successful (see also Alloin 1990). First of all, we were lucky - NGC 5548 underwent three significant outbursts during the first year of the study, and continues to undergo pronounced variations. In contrast, in the Wise Observatory project of 1988 , only 3 of about a dozen sources varied significantly during an entire observing season (see Maoz 1988). A second bit of good fortune was that the power spectrum of the continuum variations turned out to be steep, something like $1 / f^{2}$. The continuum outbursts were smooth and well resolved, which led to accurate crosscorrelation results. What this all comes down to was that the right target was selected for the study - NGC 5548 is apparently bright and well placed in the sky for a long observability window for IUE. It had been observed frequently as part of earlier projects 
(e.g., Wamsteker et al. 1990 and Peterson et al. 1990) which indicated variability time scales well suited to the capabilities of IUE.

Both the satellite-based and ground-based programs were remarkable in terms of the resources required. Overall, over 100 individuals were involved directly and are co-authors on the Clavel et al. (1990) and Peterson et al. (1991) papers. The IUE project required scheduling $\sim 60$ half-shifts at four-day intervals, and this was possible only because of the cooperative spirit between Vilspa and Goddard. The ground-based program involved about 20 telescopes from all over the northern hemisphere. The great support this project enjoyed was, I believe, because it was the right project at the right time - the idea for this effort came about at the Segovia meeting in 1987, and gained momentum at the Georgia State meeting a few weeks later. Organization of the concurrent ground-based effort began in earnest at IAU Symposium 134 the following summer. Each of these meetings featured lively debates on interpretation of spectral variations. Even though the results of the earlier studies were often inconclusive, it was obvious that there was great hope for learning about the inner structure of AGNs given enough cloeely spaced observations during periods of significant activity.

There were also three other circumstances which contributed to the success of this effort. The first of these was that IUE was a mature project. It has been an enormously successful workhorse which has more than fulfilled its original goals. At this point in its mission, it was ripe to undertake something rather innovative. Moreover, the climate for this project was right as the Europeans had been using IUE for similar albeit somewhat less intensive studies for years (which may account for what I would characterize as the early enthusiasm of ESA/SERC for the project in contrast to the early caution of NASA). The second circumstance was the relatively recent proliferation of CCD spectrographs and cameras on moderate-aperture and smaller telescopes. The ground-based results would have been far less spectacular if the typical uncertainties had turned out to be $\sim 10 \%$ or worse rather than better than $\sim 5 \%$. The third important development was in our ability to communicate rapidly. The electronic mail networks, which had come into widespread use only shortly before planning for this project began, were critical in organization and execution of the effort.

In light of the results of this project, what are the most interesting problems for future studies in emission-line variability? Certainly one of the most important questions is the radius - luminosity relationship, which is predicted to be $r \propto L^{1 / 2}$ in the simplest models. This relationship is fundamentally important in refining photoionization models, extending our understanding to high-luminosity quasars, and explaining correlations such as the Baidwin effect.

It is also important to continue studying NGC 5548 to determine if the transfer function is stationary, i.e., does either the geometry or the emissivity distribution of the BLR change with time? Netzer and Maoz (1990) have already opened this question by noting that the three individual outbursts shown in Fig. 1 have different lags.

The one issue that I find the most confusing is the relationship between the observable continuum at energies less than 1 Ryd and the high-energy continuum. X-ray variability data show different characteristics than we see at lower energies - the variations in the $\mathrm{X}$-ray are apparently faster and their power spectrum is flatter than what we see at lower energies. It is extremely important that we understand the variability in these different wavebands, since the X-rays contribute substantially to the energy input to the BLR. 
If future massive monitoring efforts are to be undertaken, I think that the most important lessons to be learned from the recent campaigns are the following:

1. The different response time for the various emission lines show that it is important that all of the lines are measured simultaneously. One cannot infer the temporal behavior of one line from the behavior of another.

2. For reasons we do not yet understand, some AGNs (e.g., NGC 5548) are "reliably" variable, i.e., the probability of observing continuum and emission-line variability over the course of one observing season is quite high. Other sources seem to vary only episodically (e.g., Akn 120). Undoubtedly, observing only the most variable AGNs will introduce some kind of selection effect, perhaps related to the inclination of the BLR or the purported accretion disk; however, at this early stage in reverberation mapping it is most important to get some return on the investment of so much observational effort. It is important to choose a source that one is quite confident will vary during the campaign.

3. We can also see from Fig. 2 that the amplitude of variation is often not very large, even though the total range in brightness can be a magnitude or more. For the lightcurve data to be useful, it is essential that the amplitude of variability greatly exceed the measurement errors. It is important that the mean measurement uncertainties are smaller than $\sim 5 \%$. Unless large telescopes are used in future campaigns, this probably restricts massive monitoring programs to apparently brighter AGNs.

\section{Acknowledgements}

I wish to thank the National Science Foundation and NASA for support of this work under grants AST-8915258 and NAG5-1366, respectively, and the organizers of this conference for their hospitality. I gratefully acknowledge travel support from the Office of Research and Graduate Studies of The Ohio State University. I also thank Gail Reichert, Jim Webb, and Mike Crenshaw for allowing me to use their recent low-state ultraviolet continuum measurement in Fig. 3 and Hagai Netzer for comments on this manuscript. 


\section{References}

Alloin, D. 1990, in Evolution in Astrophysics: IUE Astronomy in the Era of New Space Missions, ESA Publication ESA-SP-310, in press.

Blandford, R.D., and McKee, C.F. 1982, Ap. J., 255, 419.

Clavel, J., et al. 1990, Ap. J., in press.

Crenshaw, D.M., and Blackwell, J.H., Jr. 1990, Ap. J. (Letters), 358, L37.

Edelson, R.A., and Krolik, J.H. 1988, Ap. J., 333, 646.

Gaskell, C.M., and Sparke, L.S. 1986, Ap. J., 305, 175.

Horne, K., Welsh, W.F., and Peterson, B.M., 1990, preprint.

Maoz, D. 1988, in Active Galactic Nuclei, ed. D.E. Osterbrock and J.S. Miller, (Dordrecht: Kluwer), p. 100.

Netzer, H., and Maoz, D. 1990, preprint.

Peterson, B.M. 1988, Pub. A.S.P., 100, 18.

Peterson, B.M., Reichert, G.A., Korista, K.T., and Wagner, R.M. 1990, Ap. J., 352, 68.

Peterson, B.M., et al. 1991, Ap. J., in press.

Reichert, G.A., Webb, J., and Crenshaw, D.M. 1990, private communication.

Wamsteker, W., Rodríguez-Pascual, P., Wills, B., Netzer, H., Wills, D., Gilmozzi, R., Barylak, M., Talavera, A., Maoz, D., Barr, P., and Heck, A. 1990, Ap. J., 354, 446.

This book was processed by the author using the TEX macro package from Springer-Verlag. 